\title{
PERFORMANCE ANALYSIS OF ANTI-COLLISION ENABLED ROBOT USING HYBRID BEACON SCHEDULING APPROACH
}

\author{
${ }^{1}$ P. Prabakaran and ${ }^{2}$ M. Saravanan \\ ${ }^{1}$ Department of ECE, SBM College of Engineering and Technology, Dindigul, India \\ ${ }^{2} \mathrm{CR}$ Engineering College, Madurai, India
}

Received 2014-01-08; Revised 2014-01-22; Accepted 2014-03-06

\begin{abstract}
In multi-robot environment, when many robots are moving in the same workspace, there is a possibility of their physical collision with themselves as well as with physical objects. In this study, hybrid beacon scheduling protocol is proposed and for avoiding such collisions in robotic mobile environment with low latency and power consumption. The purpose of deploying this protocol is to collect relevant data for processing and reporting. In particular, based on data reporting, the robotic nodes can be classified as timedriven or event-driven. The motivation behind this research is that it dynamically switches between the eventdriven data-reporting and time-driven data-reporting schemes. As such, the proposed protocol accurately analyzes the environment being monitored using only moderate resource consumption. We have implemented the proposed protocol on a network simulator and analyzed its behaviors under various conditions.
\end{abstract}

Keywords: Robot, Hybrid Beacon Protocol, Latency

\section{INTRODUCTION}

Localization for autonomous mobile robots is the process of finding out and tracking the position and direction of the robot in any given environment. Localization has become a subject matter over the past few decades. Localization is the principle practice to allow a robot to navigate, investigate and carry out its allotted work effectively without human intrusion. Exteroceptive sensory systems, such as ultrasonic sensors, light detection and ranging devices (LRFs) and image sensors, act as alternative techniques in addressing the above mentioned restriction by offering information about the external environment around the robot. These external sensors help in determining the orientation of the robot with the measurements such as distance, angle, etc. with the beacons in the external environment. When all these external measurements are in correspondence with the same robot pose, self-localization method is applied.

During continuous movement of the robot, external measurements are obtained at different times and they do not coincide accurately with similar robot poses. Thus, self- localization method results in larger errors while calculating the robot pose. Hence to overcome this issue, most of the systems work by combining odometry values with measured values of external environment to obtain optimal evaluation of the robot poses. The most extensively used tool for this fusion technique is the Extended Kalman Filter (EKF). The EKF and its variants were used for localization in many conventional techniques. Also, the particle filter was used as an alternate means to combine odometry and external measurements, which was one of the probabilistic approaches.

The localization system used in this method employed ultrasonic sensors. Many ultrasonic Transmitters are attached to the ceiling at certain recognized locations in the global co-ordinate system. Three ultrasonic Receivers are placed equilaterally over the top of the mobile robot. We assume that the robot navigates over an indoor plane as common in most of the indoor applications. Also the $\mathrm{z}$ value of the robot and the Receivers are assumed to be in the global coordinate system.

The rest of this study is arranged as given: Section 2 analyzes a few related works in the field. Section 3 Corresponding Author: P. Prabakaran, Department of ECE, SBM College of Engineering and Technology, Dindigul, India 
explains the design principles of the proposed protocol in detail and analyzes its implementation. Section 4 presents the simulation result of our experimentation and finally, section 5 concludes our work.

\section{RELATED WORKS}

Bag et al. (2012), a processor incorporating smart algorithm for avoiding collisions with the help of RFID technology was developed and implemented it by using VHDL. The design procedure and the simulated results are very useful in designing and implementing a practical RFID system. This anti-collision enabled robot processor incorporates a smart anti-collision algorithm for avoiding collisions with mobile robots as well as with physical objects in multi robot environment. The RTL schematic view of the processor has been achieved from the test bench simulation followed by successful synthesis of the design.

Chen and Heinzelman (2007) made a survey of all routing protocols designed for real-time applications in mobile ad hoc networks. The major dispute in enabling real-time applications for mobile ad hoc networks was integrating support for Quality of Service (QoS), as such providing assured bandwidth and delay constraints.

Kumar and Sivalingam (2010) high energy efficient protocol is proposed for the wireless sensor networks and provides reduced delay of the sensor node for data transmission and reception. It does not support large number of sensor nodes throughout the network.

Sudha and Mohan (2011) presented a hardwareefficient algorithm and VLSI architecture to find a path for a mobile robot in the image of an environment captured by an overhead camera. Their algorithm calculates a distance map for identifying the collisionfree region for the robot and then builds a breadth-first search tree to find a path in that region. Thus, a path is obtained from the start point to the destination, which is also the shortest path in terms of the step count.

A probabilistic rebroadcast based on neighbor coverage was used by Zhang et al. (2012) to reduce routing overhead in nodes of mobile environment. A novel rebroadcast delay was introduced to utilize the neighbor coverage knowledge for determination of the rebroadcast order. Finally, the additional coverage ratio is obtained more accurately by sensing the neighbor coverage knowledge.

\section{PROPOSED PROTOCOL}

In this study, STORM and hybrid beacon scheduling protocol is proposed which can dynamically switch between the event-driven scheme and the time-driven scheme in robotic nodes distribution environment. The proposed protocol performs event-driven scheme, when a relevant event causes data propagation by robotic nodes. The protocol is designed such that, while the robotic nodes are detecting an event of interest, the nodes going to detect an event in the near future will be busy employed in the time-driven data reporting scheme. The robotic nodes continuously detects the event and sends data to a central point from the point of occurrence of an event to the point at which the event becomes null, thereby enabling exact analysis of the other robotic movements in their environment. A hybrid beacon scheduling protocol has been developed which is being incorporated with STORM to allow robotic nodes to dynamically switch between the event-driven data reporting scheme and the time-driven data reporting scheme depending on node context. Thus, the proposed system analyzes the environments more accurately than the event-driven data reporting scheme and uses lesser energy compared to the time-driven data reporting scheme.

The fundamental elements of the proposed hybrid beacon scheduling protocol are, Fig. 1 it can switch dynamically between the time-driven data reporting scheme and the event-driven data reporting scheme and Fig. 2 robotic nodes that will detect the events in the near future, or which are in close proximity to those nodes detecting the events, are occupied in the time-driven data reporting scheme. Moreover, these nodes inform other nodes about their changes so that neighbouring nodes continuously circulates data to other robotic nodes in their environment. Similarly, when the distance between two consecutive robots is about large with threshold, the robotic nodes switch back to the event-driven data reporting scheme, thus consuming low power when compared with other switching protocols.

The proposed algorithm possesses a threshold value, a threshold variable and two counter-variables to control the assertiveness level for changes in data-reporting schemes. The threshold value decides the event of interest to occur, i.e., as the value of the sensed attribute exceeds the threshold value, a robotic sensor node reports it to a central station. The threshold variable is the average of the values of the sensed attribute over a time interval of the robotic nodes in their mobile environment. Assume that the current data-reporting scheme of a robotic sensor node is event-driven; then a sensor node periodically computes the average of the sensed attribute over a recent time and updates the two counter-variables, $\mathrm{p}$ and $\mathrm{q}$, consequently. 


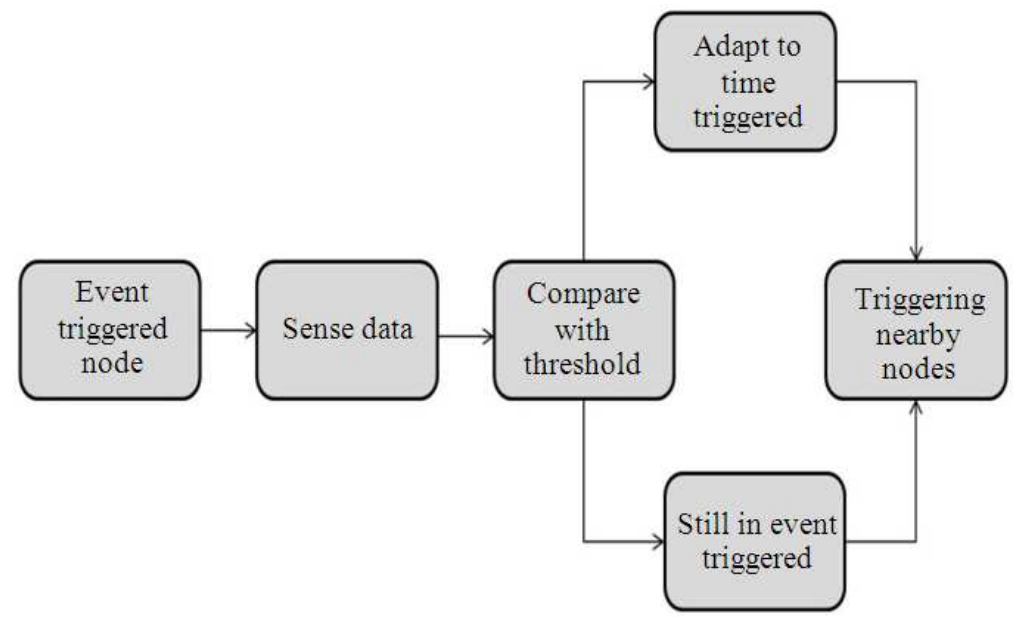

Fig. 1. Block diagram of proposed scheme

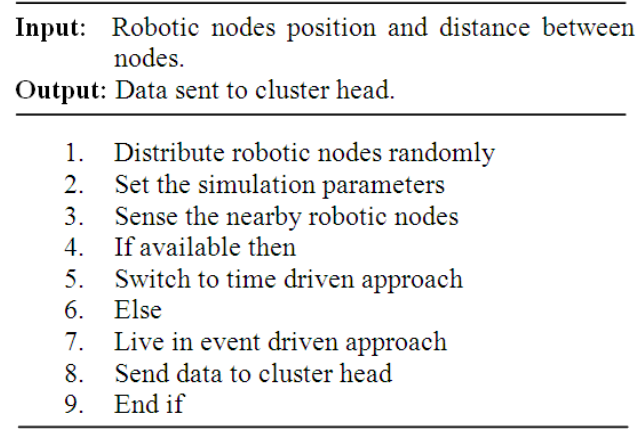

Fig. 2. Pseudo code for proposed hybrid beacon scheduling

If ( $\mathrm{p} \geq$ Pstart) or ( $\mathrm{q} \geq \mathrm{Q}$ start $)$, a sensor node switches to the time-driven data reporting scheme and reports the sensed attribute continuously over time. The average value of the sensed attribute is compared with threshold. If it lies below the threshold, then the two counter-variables are reset, thus eliminating the risk of a transient response. The data capturing an event are still transmitted to the central station without interruption.

Once a robotic sensor node switches to the timedriven data reporting scheme, it broadcasts its change to other neighbouring nodes during its continuous data transmission. The distance of a node with its neighbour node can be determined using two configurable parameters, namely, Hop Time (HT) and Valid Time (VT). HT is the number of hops within which sensor nodes must switch to the time-driven data reporting scheme. VT indicates the active time to be used by a robotic sensor node in the time driven data reporting process.
The proposed method performs well in multiple event-detection environments, i.e., when robotic nodes sense different attributes through simple modification. Consider for example, if a robotic sensor node sensed the attributes and broadcasts it to the time-driven datareporting scheme to neighbouring robotic nodes consequently. It sends the type of attribute that caused the switching, along with HT and VT, the sensor node helps its neighbouring nodes in determining the type of data needed to be collected in a time-driven approach.

\section{RESULTS}

In order to evaluate the effectiveness of our approach, we implemented the proposed protocol on Network Simulator version 2 . The simulation was performed on a network of 100 robotic nodes and a fixed base station. The nodes were placed randomly in the network and it was assumed that they change position randomly. We evaluate the performance of routing protocols using the following performance metrics-packet delivery ratio and average end-to-end delay.

\subsection{Packet Delivery Ratio}

It is the ratio of the number of data packets successfully received by the destinations to the number of data packets generated by the CBR source, i.e., the ratio between number of delivered messages and total generated messages. Delivery ratio reflects the impacts of our schemes on the performance of a robotic network with nodes. Figure 3 depicts the nodes distribution in wireless sensor networks. 
Table 1. Performance Analysis of driven approaches

\begin{tabular}{llll}
\hline Approaches & Transaction rate (b/sec) & Power consumption (joules) & Latency (sec) \\
\hline Time driven approach & 30,100 & 20.0 & 7.8 \\
Event driven approach & 31,000 & 18.0 & 7.0 \\
Hybrid driven approach & 35,000 & 15.7 & 6.6 \\
\hline
\end{tabular}

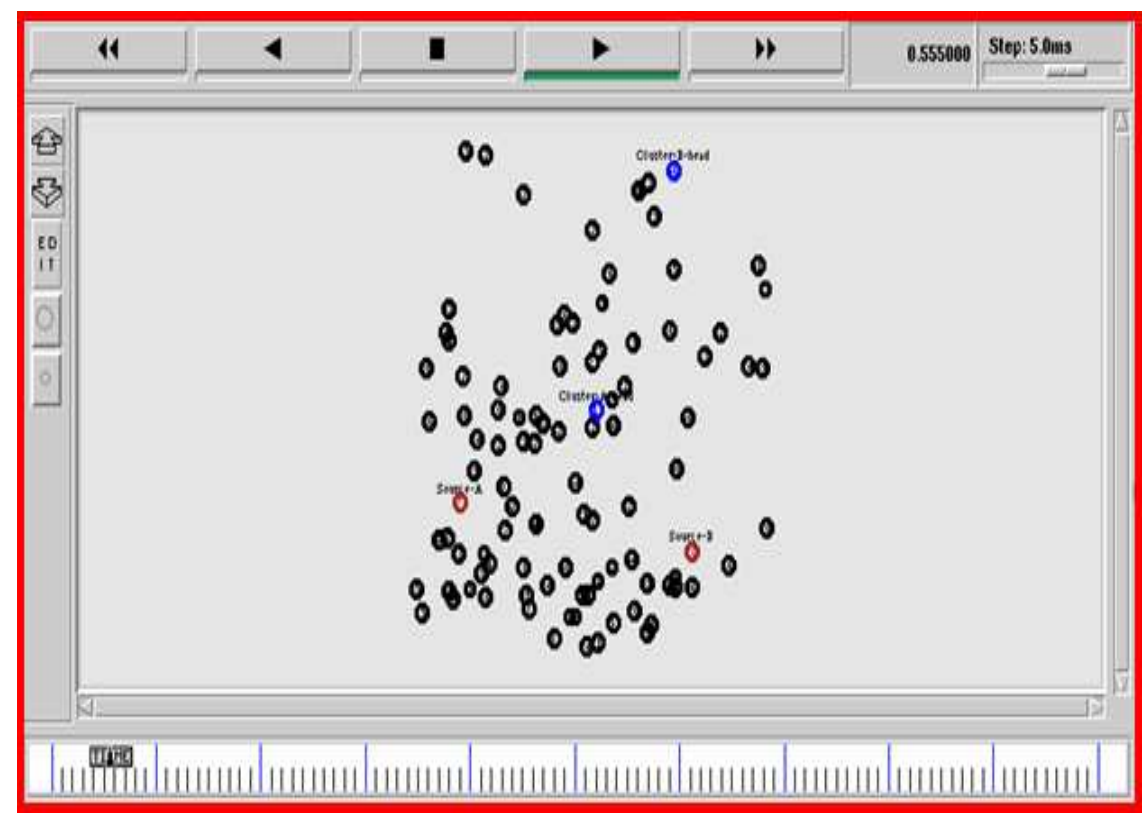

(a)

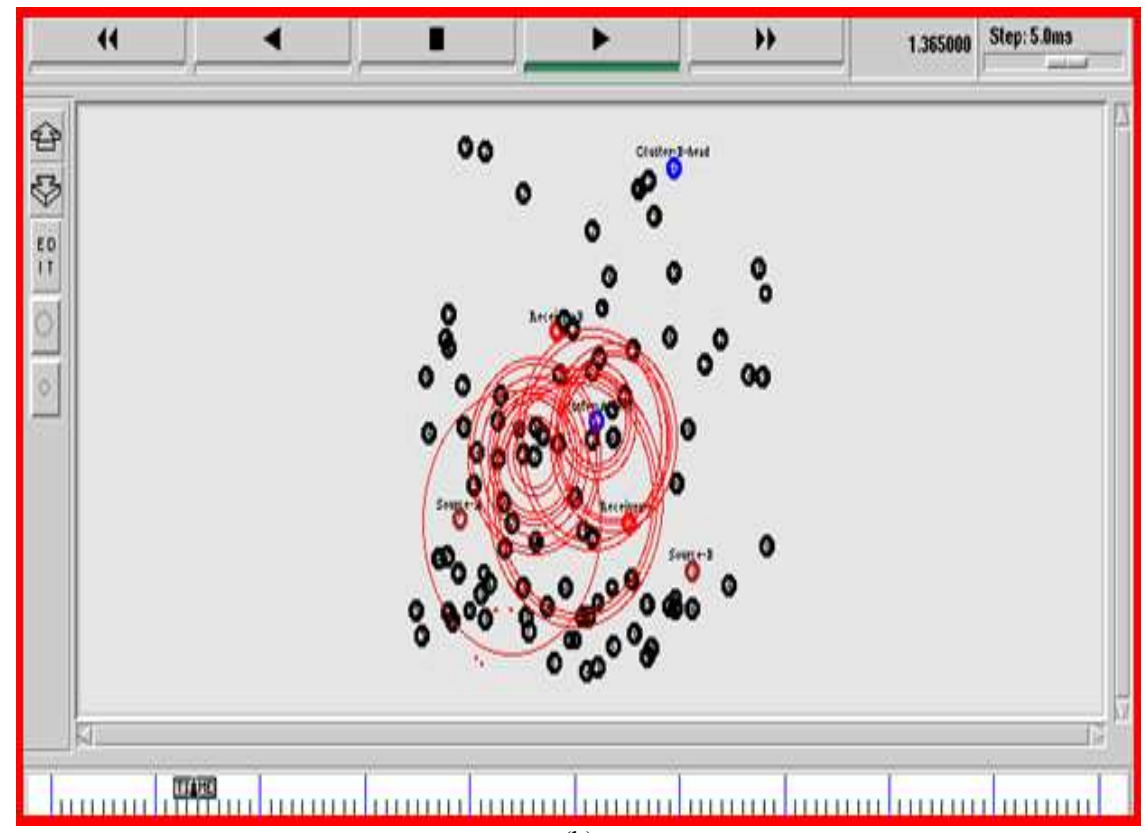

(b)

Fig. 3. (a) Robotic nodes distribution; (b) Data transmission and reception among nodes 
P. Prabakaran and M. Saravanan / American Journal of Applied Sciences 11 (5): 837-843, 2014

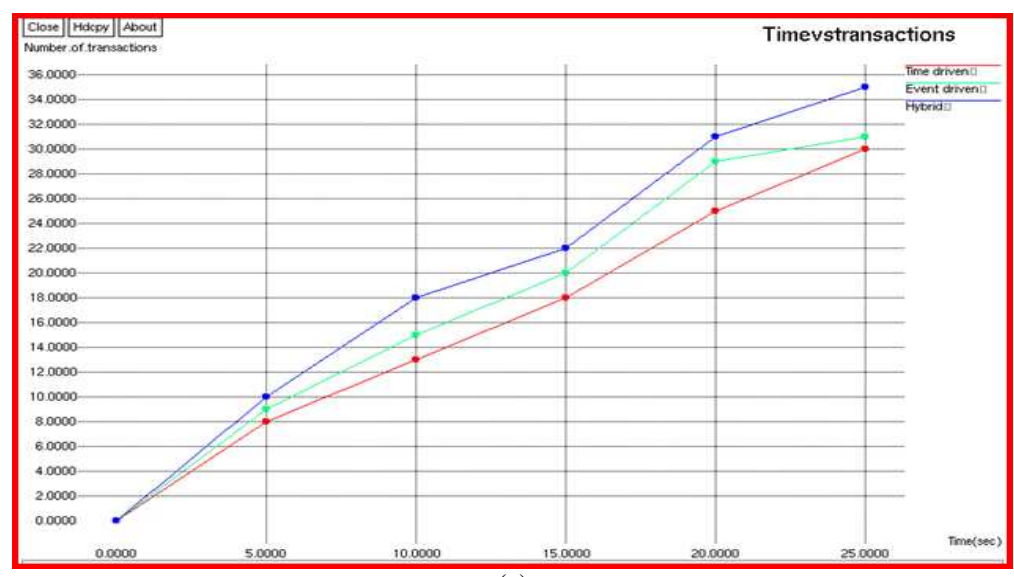

(a)

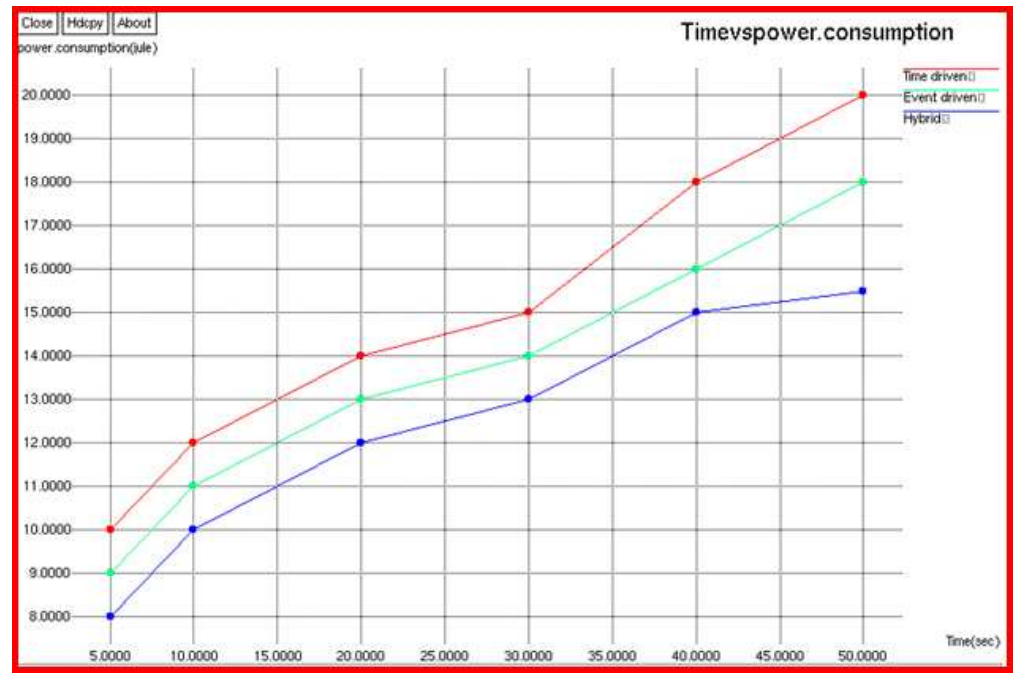

(b)

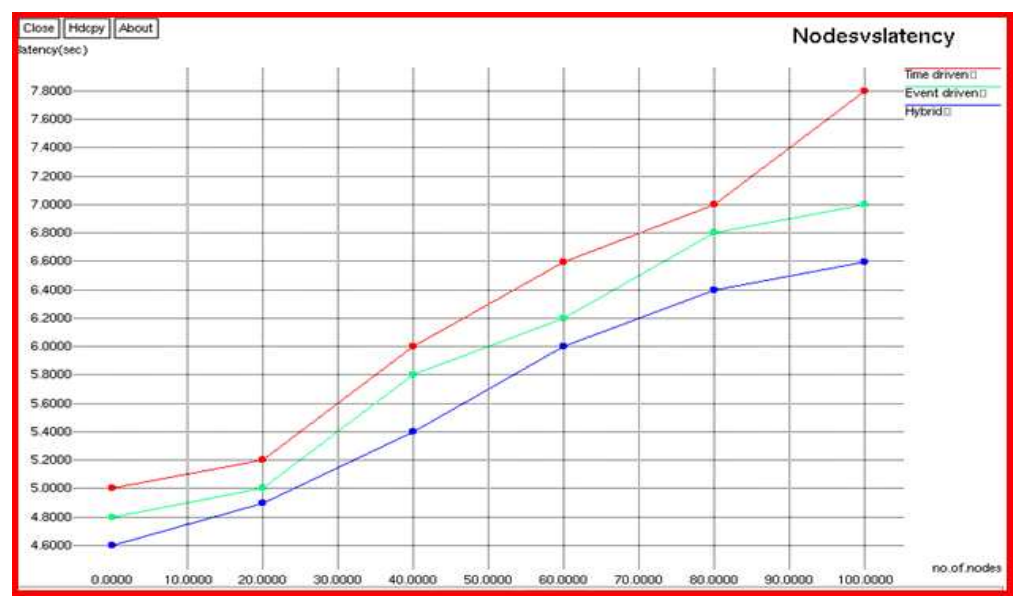

(c)

Fig. 4. Graphical illustrations (a) transactions analysis; (b) power consumption analysis; (c) latency analysis 


\subsection{Average End-to-End Delay}

It is the average delay of successfully delivered packets from source to destination node. It includes all possible delays from the sources to destinations. Figure 4 illustrates the average end-to-end delay of packets received at the destinations with increasing network density and the same is tabulated in Table 1.

We evaluate the effect of one of the following parameters on the performance of routing protocols. The number of nodes is varied from 50 to 100 in a fixed field for evaluating the effect at various network densities.

\section{DISCUSSION}

The proposed protocol has been evaluated based on the two performance parameters-packet delivery ratio and average end-to-end delay.

The proposed protocol significantly increases the packet delivery ratio as the number of collisions is reduced by the protocol considerably. Thereby, reducing the number of packet drops caused by collisions. The packet delivery ratio is extensively improved by about
$99.1 \%$ on the average by the proposed protocol on comparing it with the conventional SLPS (Li et al., 2012) and AODV (Zhang et al., 2012) protocols.

Also, if compared with the AODV protocol alone, the proposed protocol improves the packet delivery ratio by about $1.02 \%$ under the same condition.

The proposed protocol decreases the average end-to end delay due to a decrease in the number of redundant rebroadcasting packets. The redundant rebroadcast increases the delay because it incurs too many collisions and interferences, leading to excessive packet drops, thereby increasing the number of retransmissions in MAC layer, which finally increases the delay.

The redundant rebroadcast causes excessive channel contentions, thus increasing the back off timer in MAC layer, which further increases the delay. Thus, it is necessary that the redundant rebroadcast must be greatly reduced in order to decrease the delay.

Table 2 and 3 provide the data for comparison of proposed protocol with other existent protocols in terms of power consumption, latency and packet delivery ratio, etc. The same is graphically represented in Fig. 5 and 6.

Table 2. Performance comparisons with DSR protocol

\begin{tabular}{llll}
\hline Methodology & Total overhead (bits) & Latency (sec) & Power consumption (joules) \\
\hline Proposed method & 28,000 & 1.2 & 26.6 \\
DSR & 30,000 & 6.5 & 32.1 \\
\hline
\end{tabular}

Table 3. Performance Comparisons of proposed method

\begin{tabular}{llll}
\hline Methodology & Packet delivery ratio & Latency (sec) & Power consumption (joules) \\
\hline Proposed method & 99.1 & 1.2 & 26.6 \\
SLPS (Yuan et al., 2012) & 95.1 & 1.6 & 36.0 \\
AODV (Zhang et al., 2012) & 96.7 & 7.0 & 28.1 \\
\hline
\end{tabular}

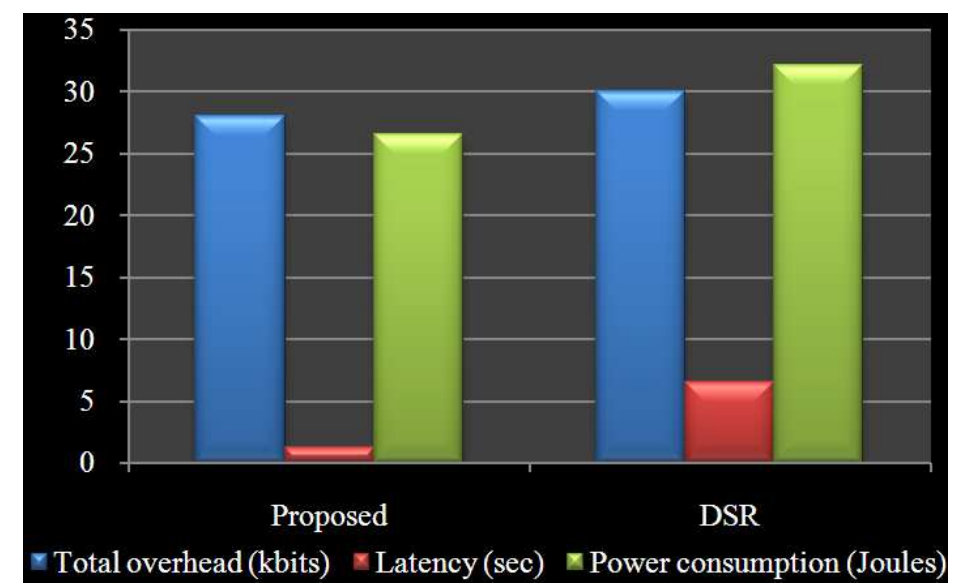

Fig. 5. Graphical plot of performance comparison of proposed method with DSR protocol 


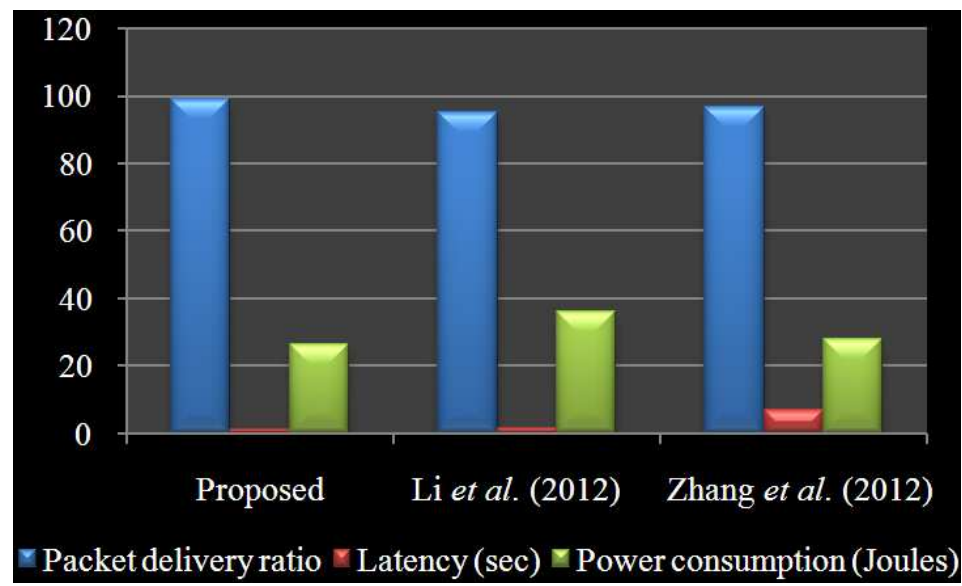

Fig. 6. Graphical plot of performance comparison of proposed method with SLPS and AODV methods

\section{CONCLUSION}

The hybrid beacon scheduling protocol is developed for avoiding collisions between mobile robot nodes in indoor robotic environment. This protocol adapts or switches between time driven and data driven schemes to reduce the power consumption and latency. The proposed protocol accurately analyzes the environment being monitored using only moderate resource consumption. The main limitation of this research in this paper is that it consumes high power and total overhead ratio, which will not suitable for real time applications. In further work, an VLSI implementation of processor which incorporates smart algorithm for avoiding such collisions will be considered.

\section{REFERENCES}

Bag, J., K.M. Rajanna and S.K. Sarkar, 2012. Design and VLSI implementation of anticollision enabled robot processor using RFID technology. Int. J. VLSI Design Commun. Syst., 3: 51-65. DOI: 10.5121/vlsic.2012.3605. 51

Chen, L. and W.B. Heinzelman, 2007. A survey of routing protocols that support QoS in mobile ad hoc networks. IEEE Netw., 21: 30-38. DOI: 10.1109/MNET.2007.4395108
Kumar, A.K. and K.M. Sivalingam, 2010. Energyefficient mobile data collection in wireless sensor networks with delay reduction using wireless communication. Proceedings of 2nd International Conference on Comm. Systems and Networks, Jan. 5-9, pp: $1-10$. DOI: 10.1109/COMSNETS.2010.5431982

Li, Y., J. Ren and J. Wu, 2012. Quantitative measurement and design of source-location privacy schemes for wireless sensor networks. IEEE Trans. Parallel Distributed Syst., 23: 1302-1311. DOI: 10.1109/TPDS.2011.260

Sudha, N. and A.R. Mohan, 2011. Hardware-efficient image-based robotic path planning in a dynamic environment and its FPGA implementation. IEEE Trans. Indust. Electron., 58: 1907-1920. DOI: 10.1109/TIE.2010.2054051

Zhang, X.M., E.B. Wang, J.J. Xia and D.K. Sung, 2012. A neighbour coverage based probabilistic rebroadcast for reducing routing overhead in mobile ad hoc networks. IEEE Trans. Mobile Comput., 11: 424-433. DOI: 10.1109/TMC.2011.277 